\title{
A Proposal of a Support System for Motivation Improvement Using Gamification
}

\author{
Kohei Otake ${ }^{1, *}$, Ryosuke Sumita ${ }^{2}$, Makoto Oka $^{3}$, Yoshihisa Shinozawa ${ }^{1}$, \\ Tomofumi Uetake ${ }^{4}$, and Akito Sakurai ${ }^{1}$ \\ ${ }^{1}$ Graduate School of Science and Technology, Keio University, 3-14-1 Hiyoshi, Kohoku-ku, \\ Yokohama-shi, Kanagawa-ken 223-8522, Japan \\ otake_kohei@a8.keio.jp, \{shino,sakurai\}@ae.keio.ac.jp \\ ${ }^{2}$ Hitachi Solutions. Ltd, Japan, 4-12-7, Higashishinagawa, \\ Shinagawa-ku, Tokyo 140-0002, Japan \\ va.poccuri@gmail.com \\ ${ }^{3}$ Faculty of Knowledge Engineering, Tokyo City University, 1-28-1 Tamazutsumi, \\ Setagaya-ku,Tokyo, 158-8557, Japan \\ moka@tcu.ac.jp \\ ${ }^{4}$ School of Business Administration, Senshu University, 2-1-1 Higashimita Tama-ku, \\ Kawasaki, 214-8580, Japan \\ uetake@isc.senshu-u.ac.jp
}

\begin{abstract}
In this research, we built a support system for improving motivation by utilizing gamification, targeting one university circle, the Senshu University Philharmonic Orchestra, as an example of voluntary communities. The purpose of this research was to maintain and improve the motives of each individual orchestra member for practice. Analysis of the current conditions clarified the following two factors as obstacles for improving the motivation of section members: "It is difficult to realize one's own practice achievements" and "Achievement level of practice of other members are unknown". In order to solve these problems, we built a system that visualized practice achievements and enabled sharing of the information among section members, with applying the concept of gamification in order to reinforce these functions. Through the experimental result, we consider that the effectiveness of this system was successfully verified.
\end{abstract}

Keywords: Gamification, Voluntary Community, Motivation Management.

\section{Introduction}

Social networking services (SNSs) such as Twitter and Facebook have attracted much attention and become widely popular recently. SNSs enable easy information sharing from remote areas in an asynchronous manner. These services not only serve to complement communication in real-world communities, but also promote work, tasks, and

\footnotetext{
* Corresponding author. 
management work within such communities. Therefore, companies, schools, and municipalities are quite actively using SNSs with the aim of revitalizing communication within communities formed in the real world, along with maintenance and improvement of motives among members. However, application of SNSs in non-profit and voluntarily-formed organizations (local communities, university circles, etc.) has found it difficult to care for the amount of website traffic and also retain active users continuously. Under present circumstances, still, potential capacity of SNSs is not fully utilized or fulfilled. These findings show that the purpose of belonging to voluntary organizations is ascribed as not for financial profit, but for a sense of satisfaction and amusement. In addition, most of the SNSs run by voluntary organizations do not have compelling force, where each individual user has different motivation. Therefore, if any of the existing SNSs targeting organizations with financial profits and some compelling force, like ordinary companies, is then used in such organizations, it would be difficult for users to maintain and improve their motivation. In order to manage users' motives effectively in voluntary organizations, users probably need to have motivation by means of certain methods that are substituted for some compelling force.

Gamification has attracted much attention recently as a method to help users to maintain and improve their motives. Gamification is defined as "to use gaming elements, such as concept, design, and mechanics of a game, for social activities or services other than the game itself." This idea became widespread after 2010, which has empirically been applied to course design in university education [2], rehabilitation activities in the medical field [3], and e-learning [4] with the aim of maintaining and improving users' motives. This application has produced beneficial effects. At the same time, however, this field is still in the sprouting stage. Only a few attempts have been made so far at verifying the effectiveness of implementing and running an SNS system.

Targeting voluntary organizations, in this research we built a system that supports users so as to maintain and improve their motives by utilizing gamification. Through the actual operation of this system, we verified the effectiveness of gamification as a motivation maintenance and improvement method. The subject of the evaluation experiment we conducted was within one of the circles approved by Senshu University, the Senshu University Philharmonic Orchestra. During a one-month experimental period, we verified the effectiveness of this proposed method.

\section{Analysis of the Current Conditions}

\subsection{Existing Support Systems for Profit-Oriented Organizations}

Use of SNSs in profit-oriented organizations with the purposes of information sharing [5], knowledge management [6], and communication support has achieved certain positive results and contributed to profit-earning activities, still leaving issues for each purpose.

In non-profit organizations, however, although SNSs are used with the purposes similar to those of profit-oriented organizations, similar achievements are not always 
guaranteed due to the differences in responsibilities and the compelling force for belonging to a community. Through this research, we focused on gamification that has attracted much attention recently as a method to support users so as to maintain and improve their motives through an SNS or groupware.

\subsection{What is Gamification?}

Gamification is defined as "to use gaming elements, such as concept, design, and mechanics of a game, for social activities or services other than the game itself." The following seven methods are included as the gamification.

1. Honorific badges or titles are given according to achievements.

2. Names and scores of competitors are displayed on a real-time basis

3. The graphic interface shows the progress of each task

4. Virtual currency is introduced to promote purchases of virtual goods.

5. Rewards such as coupons or gifts are provided

6. Assignments that encourage users to collaborate together are presented

7. Simple games are prepared between activities in order to keep users from being bored

In voluntary organizations, community members do not always maintain and improve their motives simply by financial rewards. For example, they do so by confirming their growth. As a motive that aims toward personal growth, humans have a need for esteem to be approved or commended by others [7][8], in addition to the convenience that can be acquired after growth. Incorporation of gamification in SNSs is likely to meet the need for esteem of each individual user, maintain and improve the individual motivation, and enhance the entire organizational performance.

\section{The Purpose of Our Research}

While focusing on a voluntary organization, the Senshu University Philharmonic Orchestra, the experimental subject, in this research we built and evaluated a support system for users to maintain and improve their motives based on gamification. The purpose was for each member to maintain and improve their motivation for practice. In other words, the purpose of this paper is to affirm that the introduction of this proposed system fosters an environment where community members can participate in the community ever before, which revitalizes the entire community. Our ultimate purpose of this research is to build a motivational support and improvement system that fosters a better environment for community members to participate in the community more actively than ever before, and the community itself is also revitalized, by introducing this proposed system. 


\section{Experimental Subjects}

The experimental subject, the Senshu University Philharmonic Orchestra (SUPO), is an amateur orchestra consisting of volunteer students. All the members are not the same position in this orchestra. For example, a section leader exists in each musical instrument section. As for analysis of the current conditions, in this research we conducted a questionnaire survey regarding motives of the members of SUPO in order to clarify the motivational problems that this orchestra had.

In order to analyze the current conditions, we conducted questionnaire and interview surveys regarding "Problems associated with maintenance and improvement of motives" for 31 male and female members of SUPO. As a result of the survey, the following two problems became clear.

- It is difficult to realize the benefits of self practice

- Achievement level of practice of other members are unknown

With respect to the problem of difficulty in realizing the benefits of self practice, we considered the visualization of the amount of practice. Performing technique of musical instruments is evaluated depending significantly on feelings. In other words, it is impossible to measure the performing skills of each individual player on a quantitative scale. For this reason, each individual cannot realize his/her own growth that much, and this makes it difficult for him/her to maintain and improve motivation for practice. Humans are motivated when they realize their own growth, and their motives are improved [9]. Based on this reason, we thought that the visualization of the amount of practice can probably solve this problem.

With respect to the problem of not being able to know the achievement level of practice of other members, we examined methods for sharing information regarding the achievement level of practice which could be effective and understood intuitively. During the weekdays, each individual member of SUPO practices voluntarily. This makes it difficult each of them to know the achievement level of practice of the other members. Specifically, the section leaders devote their practice time to observe each member to understand their achievement level of practice. Therefore, we thought that these problems could be solved if an SNS could show the achievement level of practice of other members in a form by which all members can obviously understand it. This means that the section members can understand the achievement level of practice of each other, and, since information sharing produces a competition, this encourages members to improve their motivation for practice. As a result, the amount of practice increases.

We here propose a system that incorporates the gamification elements into these factors, the visualization of achievement level of one's own practice and a method for the sharing of information regarding the achievement level of practice of other members, in order to maintain and improve users' motivation effectively. 


\section{Proposal of the Support System for Motivation Maintenance and Improvement}

With the analytical results of the current conditions, we propose a new system called f-simo (fortissimo) that supports users to maintain and improve their motivation for practice by visualizing the practice achievements and understanding the achievement level of practice of each section member. In order to make motivation improvement measure effective, we proposed and introduced methods incorporating gamification in f-simo.

\subsection{The Outline of Our Proposed System}

This f-simo system provides the following four functions.

1. Increase in the avatar level along with an increase in experience points

2. Graphic representation and sharing of practice time

3. Presentation of practice time rankings

4. A character (Shimosuke) grows up by all section members together

As for these functions, 1, 2, and 6 of gamification methods (Section 2.2) were incorporated. Next section will describe each of the above functions. The figure below shows the system image of f-simo.

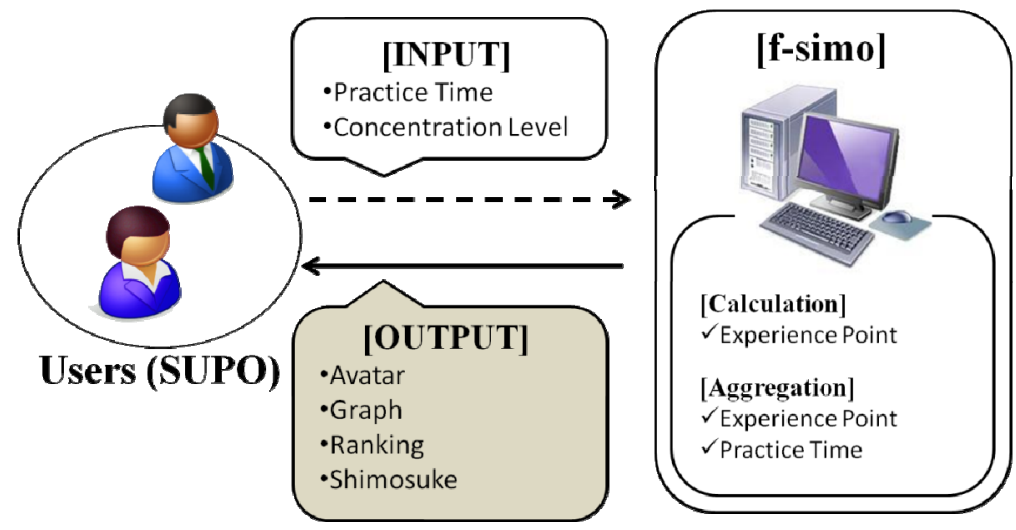

Fig. 1. System image of f-simo

After practice, on the corresponding form, the users enter their practice time and concentration level as input items regarding practice based on a self-evaluation scale of one to five. The concentration level is required to be entered because the practice achievements could depend on the concentration level even though each member spent the same period of time practicing. The experience point is calculated from these two numerical values. These items, the practice time, the concentration level, and the experience point, are also used for improvement of the avatar's level and the 
character (Shimosuke) that grows up based on the cooperation of all orchestra members. Avatars referred to by this research indicate characters of users used in the system. Levels serve as the indicator within the system. When the level is improved, the numerical value written on the level, the title, and the background color of the avatar changes. "Shimosuke" is the character that grows up by collaborative assignments of users. The section below describes each function.

\section{Improvement of the Avatar Using the Experience Points}

We set this function so that the user's avatar level increases by accumulating experience points and the avatar's title changes accordingly (Fig.2). The purpose of this function was to visualize the practice achievements not only with numerical values, but also with the title and change of color so that the user can realize his/her growth. This function was inspired by the first gamification method mentioned in 2.2, "honorific badges or titles given according to achievements."

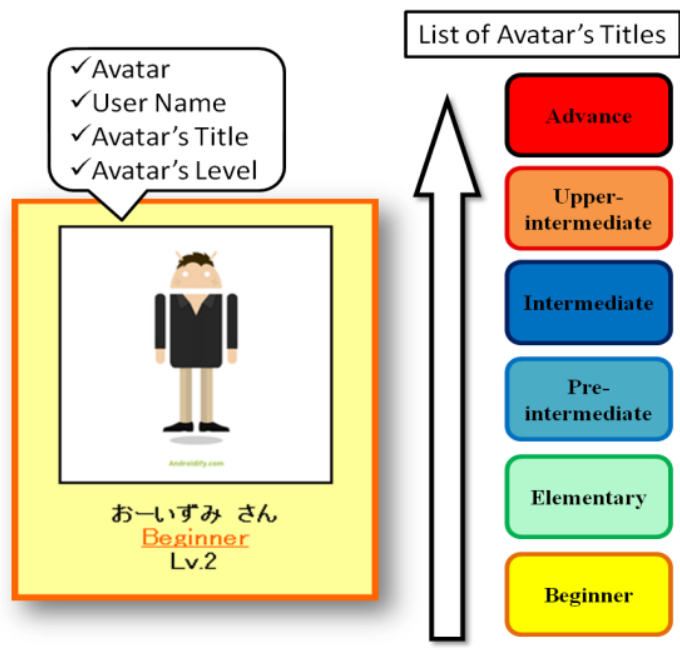

Fig. 2. The example of use and list of avatar's titles

\section{Graphical Representation of Practice Time, and Presentation and Sharing of Rankings}

We implemented the graphical representation function so that each member can understand the transition of their practice time intuitively. This function also enables users to compare their own practice time with their average practice time and the practice time of other members within the same section (Fig.3 and Fig.4). This function aims toward improving the users' motivation based on a sense of competition.

There are two rankings, the practice time of the previous day and the accumulated practice time. These two different rankings were introduced in order to avoid a decline in the motivation for practice of those who cannot come to practice on weekdays. This function was inspired by the second gamification method mentioned in 2.2, "Display of the names and scores of competitors on a real-time basis." 


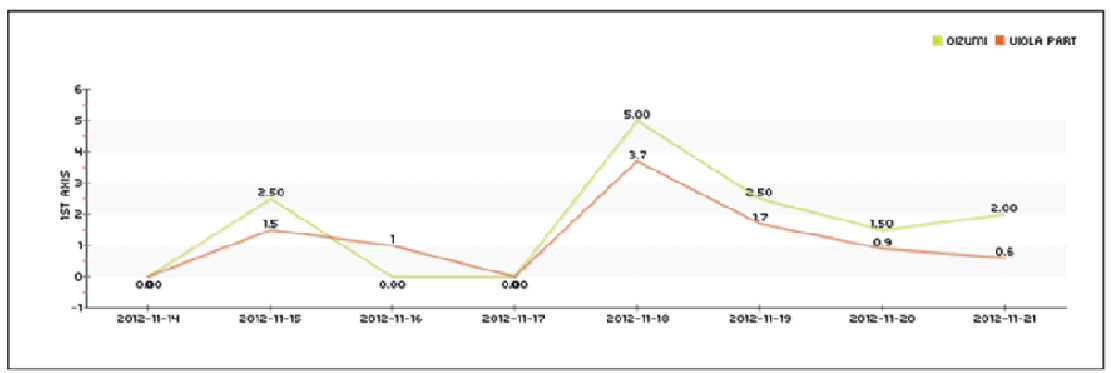

Fig. 3. A user's average practice time and the practice time of other members within "viola" section

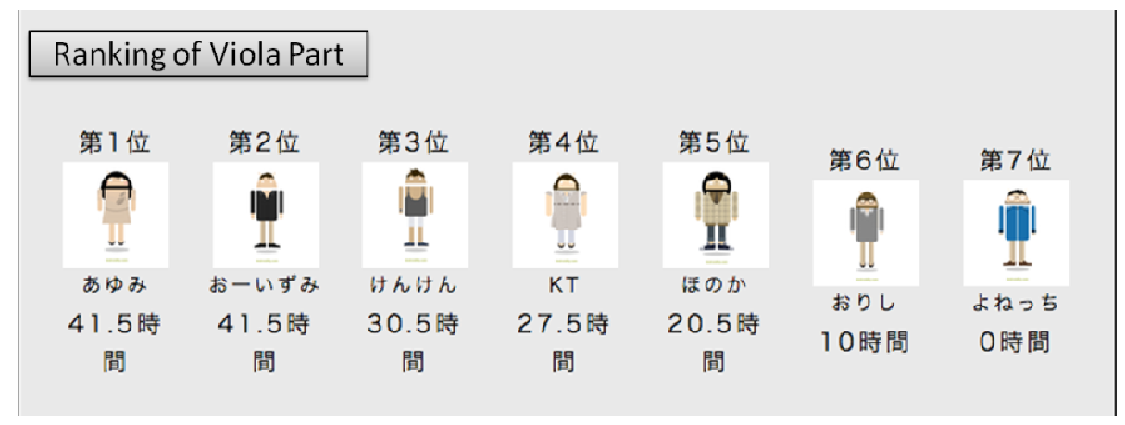

Fig. 4. The example of a graphical representation of Ranking

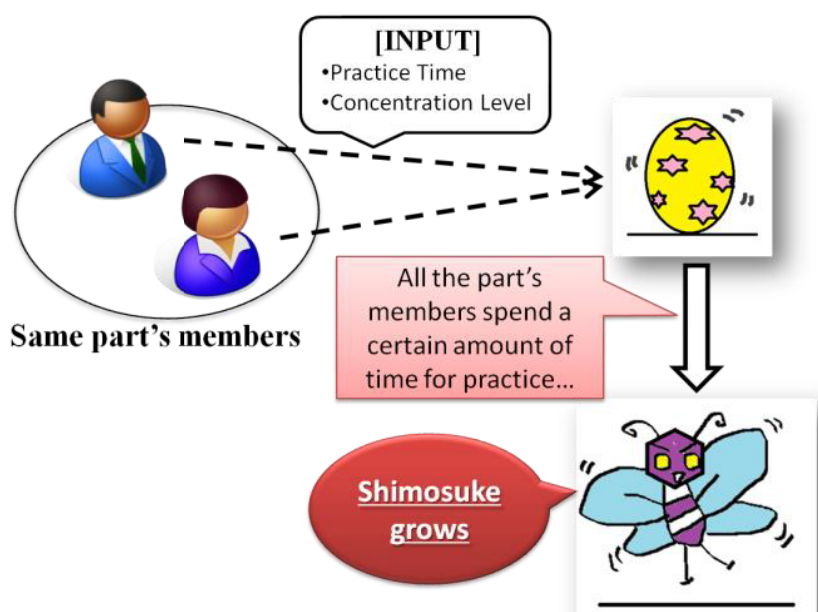

Fig. 5. The image of the cooperation which uses Shimosuke 


\section{Character (Shimosuke) that Grows up by Cooperation of All Section Members}

We implemented a character that grows when all the members of each section spend a certain amount of time for practice (Fig.5). The aim of this function was to give a common assignment for all section members to work on so that they can strengthen their group ties and improve their motivation. This function was inspired by the sixth gamification method, "Presentation of assignments for users to collaborate together."

\section{Results and Discussions}

In order to verify the effectiveness of this system, we conducted an experimental evaluation with 25 male and female SUPO members as the experimental subjects by having them use this system for one month, from November 25 to December 15 of 2012. In this verification experiment, we studied the effectiveness of this system regarding the two items:

1. Was members' motivation for practice improved?

2. Evaluations of each function

Item 1 above was determined by quantifying the number of practice sessions of members per day and the transition of practice time before and after this system was introduced (Table 1). Item 2 above were determined based on the results of the questionnaire survey conducted after the experiment (Table 2). The tables below summarize the experiment's verification results.

Table 1. Comparison of the number of practice sessions and practice time before and after the system was introduced

\begin{tabular}{|l|c|c|c|}
\hline & Before & 2nd week & 3rd week \\
\hline Number of practice sessions (times) & 0.4 & 0.6 & 0.8 \\
\hline Practice time (time) & 1.0 & 1.2 & 1.7 \\
\hline
\end{tabular}

Table 2. Evaluations for each function

\begin{tabular}{|l|c|c|}
\hline Question & $\begin{array}{l}\text { 2nd } \\
\text { week }\end{array}$ & $\begin{array}{l}\text { 3rd } \\
\text { week }\end{array}$ \\
\hline $\begin{array}{l}\text { Did the change in your avatar make you realize your growth } \\
\text { more than ever before? }\end{array}$ & 3.16 & 3.36 \\
\hline $\begin{array}{l}\text { Did graphic representation of practice time and ranking presenta- } \\
\text { tion increase your sense of competition? }\end{array}$ & 3.24 & 3.36 \\
\hline Did Shimosuke serve to strengthen the ties within your section? & 2.60 & 2.80 \\
\hline
\end{tabular}

Table 1 shows that the number of practices and practice hours increased each week. With respect to the point as to whether all section members could improve their motivation for practice, as shown by Table 2, the average number of practice sessions 
and time per day increased as the weeks went by. Since the average value before this system was introduced was exceeded, we were able to verify the effectiveness of this system. With respect to the evaluations for each function, in all functions, the value of the 3 rd week was higher than that of the 2 nd week. Moreover, we interviewed part reader after the verification experiment and we got opinion that management of the part member became easy using visualization systems.

On the other hand, a low average value was confirmed with respect as to whether Shimosuke could serve to strength the section ties. This is likely due to the short experimental period, just one month, so that the change of the character based on the collaborated assignment could slightly be confirmed. Actually at the end of the experiment, the character brought up by the members of only one of four sections was confirmed to have grown from the initial stage. The characters of the other three sections remained the same as the initial status. This point shows that the parameters related to the character growth need adjustment. When compared to the value of the 2nd week, however, the value of the 3 rd week did increase. This shows that continuous use of this function could strengthen the ties within the section.

Analysis of the access log reveals that in the third week, page views per one login increased. Analysis of the access $\log$ and the record of practice hours made it clear that the number of times of access and the practice hours are in proportion. When a user's access frequency to the site was high, the practice hours tended to increase. This tendency was notably clear in the violin part (Fig.6). We considered that competition worked strongly in the violin part, because the violin part of SUPO has many members compared with other parts.

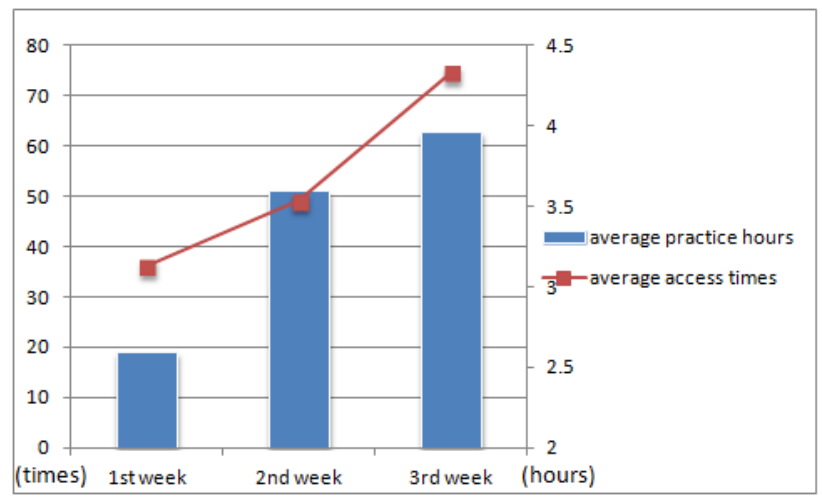

Fig. 6. Comparison of practice hours and access times in violin part

Through these experimental results, we consider that the effectiveness of this system was successfully verified. These results clarify that the following gamification methods are effective for voluntary organizations: 1. Badges obtained according to the achievement level, or level determination, and 2. Presentation of the names and scores of the current competitors on a real-time basis. When it comes to method 6 , "Assignments that promote collaborative work", the continuous use of this system could increase the effectiveness of this system to a greater extent. 


\section{Conclusion}

In this research, we built a support system for improving motivation by utilizing gamification, targeting one university circle, the Senshu University Philharmonic Orchestra, as an example of voluntary communities. The purpose of this research was to maintain and improve the motives of each individual orchestra member for practice. Analysis of the current conditions clarified the following two factors as obstacles for improving the motivation of section members: "It is difficult to realize one's own practice achievements," and "The achievement level of practice of other members are unknown." In order to solve these problems, we built a system that visualized practice achievements and enabled the sharing of information among section members, while applying the concept of gamification in order to reinforce these functions. The effectiveness verification experiment conducted for one month verified the effectiveness of this system with respect to the following functions: "1. Honorific badges or titles are given according to achievements." and "2. Names and scores of competitors are displayed on a real-time basis ". With respect to function 3, "6. Assignments that encourage users to collaborate together are presented" continuous use of this system will probably strengthen the ties between members.

We would like to put this system into continuous operation and to add another function that enhances the system loyalty. Additionally, through the use of access log obtained during this research, we are also planning to conduct further analysis focusing on user attributes.

\section{References}

1. Yano, Y., Muramoto, Y., Kitahara, K., Okubo, M.: A Proposal of SNS for activation Physical Community. Information Processing Society of Japan, 153-155 (2013) (in Japanese)

2. Kishimoto, Y., Mikami, K.: About effectiveness of university education utilizing Gamification. Journal of Digital Games Research Association Japan (2013) (in Japanese), An annual general meeting 2012, Japan

3. Matsuguma, H., Fujioka, S., Nakajima, A., Kaneko, K., Kajiwara, J., Hayashida, K., Hattori, F.: Research and Development of Serious Games to Support Stand-up Rehabilitation Exercises. Information Processing Society of Japan 53(3), 1041-1049 (2012) (in Japanese)

4. Matsumoto, T.: Possibility of e-Learning by using Gamification. Japanese Society for Information and Systems in Education 27(3), 34-40 (2012) (in Japanese)

5. Chisokukan, http://jp.fujitsu.com/group/fst/services/chisokukan/ (January 28, 2014 author checked)

6. Sabetto, T., Kotani, M.: Utilizing the enterprise social network for knowledge management. The Journal of Information Science and Technology Association 62(7), 296-301 (2012)

7. Ota, H.: Recognition and motivation [the proved effect]. Dhobun sha shuppan (2011)

8. Harvard Business Review Anthology: Power to motivate - Theory of motivation, and practice (2009)

9. Frederick, H.: Motivation to Work. John Wiley \& Sons Inc. (1959) 